\title{
Error of a temperature probe for cancer ablation monitoring caused by respiratory movements: Ex vivo and in vivo analysis
}

Camilla Cavaiola, Paola Saccomandi, Carlo Massaroni, Daniele Tosi, Francesco Giurazza, Giulia Frauenfelder, Bruno Beomonte Zobel, Francesco Maria Di Matteo, Michele Arturo Caponero, Andrea Polimadei, Emiliano Schena

- School of Engineering

\begin{abstract}
Hyperthermal techniques are spreading as an alternative to conventional surgery for cancer removal. A real-time temperature feedback can be used to adjust the treatment settings, in order to improve the clinical outcomes. In this paper, we experimentally assessed the feasibility for distributed temperature monitoring of a custom probe, which consists of a needle embedding six fiber Bragg gratings (FBGs). Since FBGs are also sensitive to strain, we focused on the analysis of the measurement error (artifact) caused by respiratory movements. We assessed the artifact both on ex vivo pig liver and lung (by mimicking the movement of these organs caused by respiration) and on in vivo trial on pig liver. Lastly, we proposed an algorithm to detect and minimize the artifact during ex vivo liver laser ablation. During both ex vivo and in vivo trials, the probe insertion within the organ was easy and safe. The artifact was significant (up to $3{ }^{\circ} \mathrm{C}$ ), but the correction algorithm allows minimizing the error. The main advantages of the proposed probe are: 1) spatially resolved temperature monitoring (in six points of the tissue by inserting a single needle) and 2) the needle is magnetic resonance (MR)-compatible, hence can be used during MR-guided procedure. Even if the model is close to humans, further trials are required to investigate the feasibility of the probe for clinical applications.
\end{abstract}

Original language $\quad$ English

Article number

7482660

Pages (from-to)

5934-5941

Number of pages

8

Journal

$\underline{\text { IEEE Sensors Journal }}$

Volume

16

Issue number

15

State

Published - Aug 12016

Cavaiola, C., Saccomandi, P., Massaroni, C., Tosi, D., Giurazza, F., Frauenfelder, G., ... Schena, E. (2016). Error of a temperature probe for cancer ablation monitoring caused by respiratory movements: Ex vivo and in vivo analysis. IEEE Sensors Journal, 16(15), 5934-5941. [7482660]. DOI: 10.1109/JSEN.2016.2574959 